\title{
RADIAL GROWTH AND EXCEPTIONAL SETS FOR CAUCHY-STIELTJES INTEGRALS
}

\author{
by D. J. HALLENBECK and T. H. MACGREGOR
}

(Received 2nd July 1992)

This paper considers the radial and nontangential growth of a function $f$ given by

$$
f(z)=\int_{|\xi|=1} \frac{1}{(1-\zeta z)^{a}} d \mu(\zeta) \text { for }|z|<1
$$

where $\alpha>0$ and $\mu$ is a complex-valued Borel measure on the unit circle. The main theorem shows how certain local conditions on $\mu$ near $e^{i \theta}$ affect the growth of $f(z)$ as $z \rightarrow e^{i \theta}$ in Stolz angles. This result leads to estimates on the nontangential growth of $f$ where exceptional sets occur having zero $\beta$-capacity.

1991 Mathematics subject classification: 30D99, 30E20.

\section{Introduction}

Let $\Delta=\{z:|z|<1\}$ and $\Gamma=\{z:|z|=1\}$. Let $\mathscr{M}$ denote the set of complex-valued Borel measures on $\Gamma$. For each $\alpha>0$ the family $\mathscr{F}_{\alpha}$ of analytic functions is defined as follows: $f \in \mathscr{F}_{\alpha}$ provided that there exists $\mu \in \mathscr{M}$ such that

$$
f(z)=\int_{\Gamma} \frac{1}{(1-\bar{\zeta} z)^{\alpha}} d \mu(\zeta)
$$

for $|z|<1$. (Here and throughout this paper every logarithm means the principal branch.)

Equation (1) is equivalent to

$$
f(z)=\int_{-\pi}^{\pi} \frac{1}{\left(1-e^{-i t} z\right)^{\alpha}} d g(t)
$$

where $g$ is a complex-valued function of bounded variation on $[-\pi, \pi]$. Throughout this paper we assume that every such $g$ is extended to $(-\infty, \infty)$ by $g(t+\pi)-g(t-\pi)=g(\pi)-g(-\pi)$. Then (2) can be rewritten

$$
f(z)=\int_{\theta-\pi}^{\theta+\pi} \frac{1}{\left(1-e^{-i t} z\right)^{a}} d g(t)
$$


for each real number $\theta$ and $g$ is of bounded variation on $[\theta-\pi, \theta+\pi]$.

We consider the effect of the differentiability of $g$, or of other local smoothness conditions at $\theta$, on the radial growth of $f$ in the direction $\theta$. In particular, this yields a new proof of the result in [2, Theorem 7] that if $f \in F_{\alpha}$ and $\alpha>1$ then $\lim _{r \rightarrow 1-}(1-r)^{a-1} f\left(r e^{l \theta}\right)=0$ for almost all $\theta$ in $[-\pi, \pi]$. If $f \in \mathscr{F}_{\alpha}$ then (1) implies

$$
|f(z)| \leqq \frac{\|\mu\|}{(1-|z|)^{\alpha}}=0\left[\frac{1}{(1-|z|)^{\alpha}}\right]
$$

and this maximal growth is achieved, for example, by

$$
f(z)=\frac{1}{(1-z)^{\circ}}
$$

It was shown in [2. Theorem 11] that if $f \in \mathscr{F}_{\alpha}$ then

$$
\left|f\left(r e^{i \theta}\right)\right|=o\left[\frac{1}{(1-r)^{a}}\right]
$$

as $r \rightarrow 1-$ except possibly for a set in $\theta$ which is countable. Also, when $\alpha>1$ any growth smaller than

$$
o\left[\frac{1}{(1-r)^{\alpha-1}}\right]
$$

is achievable for some $f \in \mathscr{F}_{\alpha}$ and for all $\theta$ [2, Theorem 8]. Thus the growths

$$
o\left[\frac{1}{(1-r)^{\alpha}}\right] \text { and } o\left[\frac{1}{(1-r)^{\alpha-1}}\right]
$$

provide extreme cases for questions concerning exceptional sets.

Theorem 2 in this paper shows that certain growths between these two extremes are associated with exceptional sets whose $\beta$-capacity is zero. For example, it is proved that if $f \in \mathscr{F}_{a}, 0<\beta<\alpha$ and $\beta<1$ then

$$
f\left(r e^{i \theta}\right)=o\left[\frac{1}{(1-r)^{\alpha-\beta}}\right]
$$

for all $\theta$ in $[-\pi, \pi]$ except possibly for a set whose $\beta$-capacity is zero.

The results obtained about radial growths are proved in more generality and are 
expressed in terms of suitable nontangential limits. Suppose that $-\pi \leqq \theta \leqq \pi$ and $0 \leqq \gamma \leqq \pi$. Let $S(\theta, \gamma)$ denote the closed Stolz angle having vertex $e^{i \theta}$ and opening $\gamma$. There are positive constants $A$ and $B$ depending only on $\gamma$ such that if $z=r e^{i \phi} \in S(\theta, \gamma)$ (and $\phi$ is chosen suitably) then

$$
\left|z-e^{i \theta}\right| \leqq A(1-|z|)
$$

and

$$
|\phi-\theta| \leqq B(1-|z|) .
$$

A function $f$ defined in $\Delta$ is said to have a nontangential limit at $e^{i \theta}$ provided that

$$
\lim _{\substack{z \rightarrow e^{i \theta} \\ z \in S(\theta, \gamma)}} f(z) \text { exists for every } \quad \gamma(0 \leqq \gamma<\pi)
$$

The results in this paper complement facts about nontangential limits proved in [3]. In particular, it was shown in [3, Theorem 5] that if $f \in \mathscr{F}_{\alpha}$ for some $\alpha$ where $0<\alpha<1$ then $f$ has a nontangential limit at $e^{i \theta}$ except possibly for a set of $\theta$ in $[-\pi, \pi]$ whose $\alpha$-capacity is zero. The focus of this paper is primarily on the growth of $f$ where $f \in \mathscr{F}_{\alpha}$ and $\alpha \geqq 1$.

\section{Radial growth and exceptional sets of zero $\beta$-capacity}

Theorem 1. Let $\alpha>0$ and for

$$
|z|<1 \quad \text { let } \quad f(z)=\int_{-\pi}^{\pi} \frac{1}{\left(1-e^{-i t} z\right)^{\alpha}} d g(t)
$$

where $g$ is a complex-valued function of bounded variation on $[-\pi, \pi]$.

(a) Suppose that $|g(t)-g(\theta)|=\alpha\left(|t-\theta|^{\beta}\right)$ as $t \rightarrow \theta$, for some $\theta$ in $[-\pi, \pi]$ and for some $\beta>0$. If $\beta<\alpha$ then $\left(1-e^{-i \theta} z\right)^{\alpha-\beta} f(z)$ has the nontangential limit zero at $e^{i \theta}$. If $\beta=\alpha$ then

$$
\frac{f(z)}{\log \left(\frac{1}{1-e^{-i \theta} z}\right)}
$$

has the nontangential limit zero at $e^{i \theta}$.

(b) Suppose that 


$$
|g(t)-g(\theta)|=o\left[\frac{1}{\log \frac{1}{|t-\theta|}}\right] \text { as } t \rightarrow \theta
$$

for some $\theta$ in $[-\pi, \pi]$. Then

$$
\left[\left(1-e^{-i \theta}\right)^{\alpha} \log \left(\frac{1}{1-e^{-i \theta} z}\right)\right] f(z)
$$

has the nontangential limit zero at $e^{i \theta}$.

Proof. Equation (3) implies

$$
f(z)=\int_{\theta-\pi}^{\theta+\pi} \frac{1}{\left(1-e^{-i t} z\right)^{\alpha}} d[g(t)-g(\theta)]
$$

and an integration by parts gives

$$
f(z)=\frac{g(\theta+\pi)-g(\theta-\pi)}{\left(1+e^{-i \theta} z\right)^{\alpha}}+i \alpha \int_{\theta-\pi}^{\theta+\pi} K\left(e^{-i t} z\right)[g(t)-g(\theta)] d t
$$

where

$$
K(z)=\frac{z}{(1-z)^{\alpha+1}}
$$

Let $0 \leqq \gamma<\pi$. If $z \in S(\theta, \gamma)$ then

$$
|f(z)| \leqq C_{1}+\alpha \int_{\theta-\pi}^{\theta+\pi} \frac{|g(t)-g(\theta)|}{\left|1-e^{-i t} z\right|^{\alpha+1}} d t
$$

where

$$
C_{1}=|g(\theta+\pi)-g(\theta-\pi)| \sup _{z \in S(\theta, \gamma)}\left\{\frac{1}{\left|1+e^{-i \theta} z\right|^{\alpha}}\right\}<+\infty
$$

This can be written

$$
|f(z)| \leqq C_{1}+\alpha \int_{-\pi}^{\pi} \frac{|g(\theta+t)-g(\theta)|}{\left|1-e^{-i(\theta+t)} z\right|^{\alpha+1}} d t
$$


Assume that $\beta>0$ and $|g(t)-g(\theta)|=o\left(|t-\theta|^{\beta}\right)$ as $t \rightarrow \theta$. Let $\varepsilon>0$. There exists $\delta$ such that $0<\delta<1$ and $|g(\theta+t)-g(\theta)| \leqq \varepsilon|t|^{\beta}$ for $|t|<\delta$. Let $A$ and $B$ be the constants described by (4) and (5) and let $r=|z|$. There exists $\eta$ such that $0<\eta \leqq 1 / 2$ and if $z \in S(\theta, \gamma)$ and $\left|z-e^{i \theta}\right|<\eta$ then $2 B(1-r)<\delta$. For $|t| \leqq \pi$ and $|z|<1$ let

$$
G(t, z)=\frac{|g(\theta+t)-g(\theta)|}{\left|1-e^{-i(\theta+t)} z\right|^{\alpha+1}} \text {. }
$$

For $1 \leqq n \leqq 5$ let $J_{n}=\int_{I_{n}} G(t, z) d t$ where

$$
\begin{aligned}
& I_{1}=[-\pi,-\delta], I_{2}=[-\delta,-2 B(1-r)], I_{3}=[-2 B(1-r), 2 B(1-r)], \\
& I_{4}=[2 B(1-r), \delta], \text { and } I_{5}=[\delta, \pi] .
\end{aligned}
$$

Clearly $G(t, z)$ is bounded for $t \in I_{1}$ and $z \in S(\theta, \gamma)$ and hence there is a constant $C_{2}$ such that $J_{1} \leqq C_{2}$ for $z \in S(\theta, \gamma)$. Likewise $J_{5} \leqq C_{3}$ for $z \in S(\theta, \gamma)$ where $C_{3}$ is some constant. For $z \in S(\theta, \gamma)$ and $\left|z-e^{i \theta}\right|<\eta$ we have

$$
\begin{aligned}
J_{2}= & \int_{-\delta}^{-2 B(1-r)} \frac{|g(\theta+t)-g(\theta)|}{\left|1-e^{-i(\theta+t)} z\right|^{\alpha+1}} d t \leqq\left(\frac{\pi}{\sqrt{2}}\right)^{\alpha+1} \varepsilon \int_{-\delta}^{-2 B(1-r)} \frac{|t|^{\beta}}{|t-(\phi-\theta)|^{\alpha+1}} d t \\
& \leqq 2^{\beta}\left(\frac{\pi}{\sqrt{2}}\right)^{\alpha+1} \varepsilon \int_{-\delta}^{-2 B(1-r)} \frac{1}{|t-(\phi-\theta)|^{\alpha-\beta+1}} d t .
\end{aligned}
$$

Suppose that $\beta<\alpha$. Then

$$
\begin{gathered}
\int_{-\delta}^{-2 B(1-r)} \frac{1}{|t-(\phi-\theta)|^{\alpha-\beta+1}} d t=\frac{1}{\alpha-\beta}\left\{\frac{1}{[\phi-\theta+2 B(1-r)]^{\alpha-\beta}}-\frac{1}{[\phi-\theta+\delta]^{\alpha-\beta}}\right\} \\
\leqq \frac{1}{(\alpha-\beta)[\phi-\theta+2 B(1-r)]^{\alpha-\beta}} \leqq \frac{1}{(\alpha-\beta) B^{\alpha-\beta}(1-r)^{\alpha-\beta}},
\end{gathered}
$$

because of (5). Hence

$$
J_{2} \leqq \frac{(2 B)^{\beta}}{\alpha-\beta}\left(\frac{\pi}{\sqrt{2}}\right)^{\alpha+1} \varepsilon \frac{1}{(1-r)^{\alpha-\beta}}
$$

The same inequality holds for $\boldsymbol{J}_{\mathbf{4}}$. Also,

$$
J_{3}=\int_{-2 B(1-r)}^{2 B(1-r)} \frac{|g(\theta+t)-g(\theta)|}{\left|1-e^{-i(\theta+t)} z\right|^{\alpha+1}} d t \leqq \int_{-2 B(1-r)}^{2 B(1-r)} \frac{\varepsilon|t|^{\beta}}{(1-r)^{\alpha+1}} d t=\frac{2(2 B)^{\beta+1}}{\beta+1} \frac{\varepsilon}{(1-r)^{\alpha-\beta}} .
$$


Since (6) implies $|f(z)| \leqq C_{1}+\alpha \sum_{n=1}^{5} J_{n}$ the estimates above yield

$$
|f(z)| \leqq C_{4}+\frac{C_{5} \varepsilon}{(1-r)^{a-\beta}}
$$

for $z \in S(\theta, \gamma)$ and $\left|z-e^{i \theta}\right|<\eta$, where $C_{4}$ and $C_{5}$ are constants. This inequality and (4) imply that

$$
\lim _{\substack{z \rightarrow e^{i \theta} \\ z \in S(\theta, y)}}\left(1-e^{-i \theta} z\right)^{\alpha-\beta} f(z)=0
$$

This proves (a) in the case $\beta<\alpha$.

Next suppose that $\beta=\alpha$. Then the estimate for $J_{2}$ given above becomes

$$
J_{2} \leqq 2^{(\alpha-1) / 2} \pi^{\alpha+1} \varepsilon \int_{-\delta}^{-2 B(1-r)} \frac{1}{|t-(\phi-\theta)|} d t
$$

By considering the cases $\phi<\theta, \phi=\theta$ and $\phi>\theta$ we find that, in general,

$$
\int_{-\delta}^{-2 B(1-r)} \frac{1}{|t-(\phi-\theta)|} d t \leqq \log \left[\frac{1}{B(1-r)}\right]
$$

Hence

$$
J_{2} \leqq 2^{(\alpha-1) / 2} \pi^{\alpha+1} \varepsilon \log \left[\frac{1}{B(1-r)}\right]
$$

The same inequality holds for $J_{4}$. Also,

$$
J_{3} \leqq \frac{2(2 B)^{a+1}}{\alpha+1} \varepsilon
$$

This yields

$$
|f(z)| \leqq C_{6}+C_{7} \varepsilon \log \left(\frac{1}{1-r}\right)
$$

for $z \in S(\theta, \gamma)$ and $\left|z-e^{i \theta}\right|<\eta$, where $C_{6}$ and $C_{7}$ are constants. Therefore 


$$
\lim _{\substack{z \rightarrow e^{i \theta} \\ z \in S(\theta, r)}} \frac{f(z)}{\log \left(\frac{1}{1-r}\right)}=0
$$

We are required to prove that

$$
\lim _{\substack{z \rightarrow e^{i \theta} \\ z \in S(\theta, y)}} \frac{f(z)}{\log \left(\frac{1}{1-e^{-i \theta} z}\right)}=0
$$

Hence if suffices to show that

$$
M \equiv \sup _{\substack{z \in S(\theta, y) \\|z|=r \\ 0 \leqq r<1}} \frac{\log \left(\frac{1}{1-r}\right)}{\left|\log \left(\frac{1}{1-e^{-i \theta} z}\right)\right|}<+\infty
$$

Inequality $(7)$ follows if it is shown that

$$
\frac{\log \left(\frac{1}{1-r}\right)}{\log \left(\frac{1}{1-e^{-i \theta} z}\right)}
$$

is bounded for $z \in S(\theta, \gamma),|z|=r$ and $r$ sufficiently near 1 . If $z=r e^{i \phi} \in S(\theta, \gamma)$ then (5) gives $|\phi-\theta| \leqq B(1-r)$ and hence there exists $r_{1}$ such that $0<r_{1}<1$ and $1-\cos (\phi-\theta) \leqq(\phi-\theta)^{2}$ for $r_{1} \leqq r<1$. Thus

$$
\begin{aligned}
\left|1-e^{-i \theta} z\right|^{2}= & (1-r)^{2}+2 r[1-\cos (\phi-\theta)] \leqq(1-r)^{2} \\
& +2(\phi-\theta)^{2} \leqq\left(1+2 B^{2}\right)(1-r)^{2}
\end{aligned}
$$

Hence

$$
\frac{1}{\left|1-e^{-i \theta} z\right|} \geqq \frac{1}{C(1-r)} \text { for } \quad r_{1} \leqq r<1, \text { where } C=\sqrt{1+2 B^{2}}
$$

There exists $r_{2}$ such that 


$$
r_{1} \leqq r_{2}<1 \text { and } \frac{1}{C(1-r)}>1 \text { for } r_{2} \leqq r<1
$$

Then

$$
\left|\log \frac{1}{\left(1-e^{-i \theta} z\right)}\right| \geqq\left|\operatorname{Re} \log \frac{1}{\left(1-e^{-i \theta} z\right)}\right|=\left|\log \frac{1}{\left|\left(1-e^{-i \theta} z\right)\right|}\right| \geqq \log \frac{1}{C(1-r)} \text { for } \quad r_{2} \leqq r<1
$$

Therefore, if $z \in S(\theta, \gamma)$ and $r_{2} \leqq r<1$ then

$$
\left|\frac{\log \frac{1}{1-r}}{\log \frac{1}{1-e^{-i \theta} z}}\right| \leqq \frac{\log \frac{1}{1-r}}{\log \frac{1}{C}+\log \frac{1}{1-r}} \equiv \sigma(r)
$$

Since $\lim _{r \rightarrow 1}-\sigma(r)=1$ this proves (7). Hence the proof of (a) is complete.

The proof of (b) follows in a similar way. We have $0<\delta<1$ and

$$
|g(\theta+t)-g(\theta)| \leqq \frac{\varepsilon}{\log \frac{1}{|t|}} \text { for }|t|<\delta
$$

The estimate on $J_{2}$ becomes

$$
\begin{aligned}
J_{2} & \leqq\left(\frac{\pi}{\sqrt{2}}\right)^{\alpha+1} \varepsilon \int_{-\delta}^{-2 B(1-r)} \frac{1}{\left(\log \frac{1}{|t|}\right)|t-(\phi-\theta)|^{\alpha+1}} d t \\
& \leqq\left(\frac{\pi}{\sqrt{2}}\right)^{\alpha+1} 2^{\alpha+1} \varepsilon \int_{-\delta}^{-2 B(1-r)} \frac{1}{\left(\log \frac{1}{|t|}\right)|t|^{a+1}} d t \\
& =\left(\frac{\pi}{\sqrt{2}}\right)^{\alpha+1} 2^{\alpha+1} \varepsilon L(r),
\end{aligned}
$$

where 


$$
L(r)=\int_{2 B(1-r)}^{\delta} \frac{1}{t^{\alpha+1} \log \frac{1}{t}} d t .
$$

Let

$$
M(r)=\frac{1}{(1-r)^{\alpha} \log \left(\frac{1}{1-r}\right)} \text { for } 0<r<1
$$

Then $\lim _{r \rightarrow 1-} L(r)=+\infty$ and $\lim _{r \rightarrow 1-} M(r)=+\infty$ and l'Hospital's rule yields

$$
\lim _{r \rightarrow 1_{-}^{-}} \frac{L(r)}{M(r)}=\frac{1}{\alpha(2 B)^{\alpha}}
$$

Hence then exists $r_{1}$ such that $0<r_{1}<1$ and

$$
\frac{L(r)}{M(r)} \leqq \frac{2}{\alpha(2 B)^{\alpha}} \quad \text { for } \quad r_{1} \leqq r<1
$$

Therefore there is $\eta^{\prime}$ such that $0<\eta^{\prime} \leqq \eta$ and

$$
J_{2} \leqq \frac{C_{8} \varepsilon}{(1-r)^{\alpha} \log \left(\frac{1}{1-r}\right)} \text { for } z \in S(\theta, \gamma)
$$

and $\left|z-e^{i \theta}\right|<\eta^{\prime}$, where $C_{8}$ is a constant. The same inequality holds for $J_{4}$.

Also

$$
\begin{aligned}
J_{3} \leqq & \frac{\varepsilon}{(1-r)^{\alpha+1}} \int_{-2 B(1-r)}^{2 B(1-r)} \frac{1}{\log \frac{1}{|t|}} d t=\frac{2 \varepsilon}{(1-r)^{\alpha+1}} \int_{0}^{2 B(1-r)} \frac{1}{\log \frac{1}{t}} d t \\
& \leqq \frac{2 \varepsilon}{(1-r)^{\alpha+1}}\left\{\frac{1}{\log \frac{1}{2 B(1-r)}}[2 B(1-r)]\right\}=\frac{4 B \varepsilon}{(1-r)^{\alpha} \log \frac{1}{2 B(1-r)}} .
\end{aligned}
$$

These estimates imply 


$$
|f(z)| \leqq C_{9}+\frac{C_{10} \varepsilon}{(1-r)^{\alpha} \log \left(\frac{1}{1-r}\right)} \text { for } z \in S(\theta, \gamma)
$$

and $\left|z-e^{i \theta}\right|<\eta^{\prime}$, where $C_{9}$ and $C_{10}$ are constants. This proves that

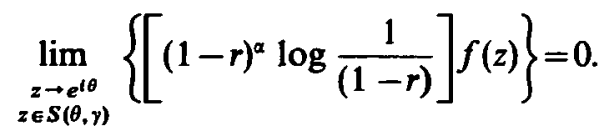

We have

$$
\left|\log \frac{1}{\left(1-e^{-i \theta} z\right)}\right| \leqq\left|\log \frac{1}{(1-|z|)}\right|+\frac{\pi}{2} \leqq 2 \log \left(\frac{1}{1-r}\right) \text { for }|z|=r \geqq r_{0}
$$

for a suitable $r_{0}\left(0<r_{0}<1\right)$. Thus (4) and (8) imply

$$
\lim _{\substack{z \rightarrow e^{(\theta \theta} z \in S(\theta, y) \\ z \in}}\left\{\left[\left(1-e^{-i \theta} z\right)^{\alpha} \log \left(\frac{1}{\left(1-e^{-i \theta} z\right)}\right)\right] f(z)\right\}=0
$$

This completes the proof of (b).

The notion of zero $\beta$-capacity for Borel subsets of $[-\pi, \pi]$ provides a useful measure of the fineness of exceptional sets for the radial growth of functions in $\mathscr{F}_{\alpha}$. The definition and properties of $\beta$-capacity are given in [1]. We note that 0 -capacity corresponds to logarithmic capacity and that if the $\beta$-capacity of a set is zero for some $\beta(0 \leqq \beta<1)$ then its Lebesgue measure is zero.

The next theorem uses the following lemma. It is proved in [4, Lemma 1$]$ in the case $0<\beta<1$ and a similar argument proves the second assertion.

Lemma 1. Suppose that $g$ is a nondecreasing function on $[-\pi, \pi]$. If $0<\beta<1$ then $|g(t)-g(\theta)|=o\left(|t-\theta|^{\beta}\right)$ as $t \rightarrow \theta$ for all $\theta$ in $[-\pi, \pi]$ except possibly for a set whose $\beta$-capacity is zero. Also,

$$
|g(t)-g(\theta)|=o\left[\frac{1}{\log \frac{1}{|t-\theta|}}\right] \text { as } t \rightarrow \theta \text { for all } \theta \text { in }[-\pi, \pi]
$$

except possibly for a set whose logarithmic capacity is zero.

Theorem 2. Suppose that $\alpha>0$ and $f \in \mathscr{F}_{\alpha}$. If $0<\beta<1$ and $\beta<\alpha$ then 
$\left(1-e^{-i \theta} z\right)^{\alpha-\beta} f(z)$ has the nontangential limit zero at $e^{i \theta}$ for all $\theta$ in $[-\pi, \pi]$ except possibly for a set whose $\beta$-capacity is zero. Also,

$$
\left[\left(1-e^{-i \theta} z\right)^{\alpha} \log \frac{1}{\left(1-e^{-i \theta} z\right)}\right] f(z)
$$

has the notangential limit zero at $e^{i \theta}$ for all $\theta$ in $[-\pi, \pi]$ except possibly for a set whose logarithmic capacity is zero.

Proof. This is a direct consequence of Lemma 1 and Theorem 1.

\section{Radial growth and differentiability}

Theorem 3 below gives estimates on the radial growth of a function $f \in \mathscr{F}_{\alpha}$ in the direction $\theta$ when the function $g$ representing $f$ is differentiable at $\theta$. The result is expressed in terms of suitable nontangential limits. This yields a new proof of a result in [2] about the radial growth of $f$ off exceptional sets of measure zero. Also it is shown that Theorem 3 is sharp when $1 \leqq \alpha \leqq 2$.

Theorem 3. Suppose that $\alpha \geqq 1, g$ is a complex-valued function of bounded variation on $[-\pi, \pi]$ and let

$$
f(z)=\int_{-\pi}^{\pi} \frac{1}{\left(1-e^{-i t} z\right)^{\alpha}} d g(t)
$$

for $|z|<1$. Assume that $g$ is differentiable at some $\theta$ in $[-\pi, \pi]$. If $\alpha>1$ then $\left(1-e^{-i \theta} z\right)^{\alpha-1} f(z)$ has the nontangential limit zero at $e^{i \theta}$. If $\alpha=1$ then

$$
\frac{f(z)}{\log \frac{1}{\left(1-e^{-i \theta} z\right)}}
$$

has the nontangential limit zero at $e^{i \theta}$.

Proof. Suppose that (9) defines $f$ where $g$ is of bounded variation on $[-\pi, \pi]$ and assume that $g$ is differentiable at $\theta$. Define the function $\tilde{g}$ by $\tilde{g}(t)=g(t)-\operatorname{tg}^{\prime}(\theta)$ for $-\pi \leqq t \leqq \pi$. Since $g^{\prime}(\theta)$ exists, we have $|\tilde{g}(t)-\tilde{g}(\theta)|=o(|t-\theta|)$ as $t \rightarrow \theta$. Also, because

$$
\int_{-\pi}^{\pi} \frac{1}{\left(1-e^{-i t} z\right)^{a}} d t=2 \pi
$$

it follows that $f(z)=f(z)+2 \pi g^{\prime}(\theta)$ where 


$$
f(z)=\int_{-\pi}^{\pi} \frac{1}{\left(1-e^{-i t} z\right)^{\alpha}} d \tilde{g}(t) \text { for }|z|<1
$$

Hence $f$ satisfies the assumptions of Theorem 1 and part (a) of that theorem where $\beta=1$ yields the conclusions.

The following theorem was proved in [2, Theorem 7] using a different argument.

Theorem 4. If $\alpha>1$ and $f \in \mathscr{F}_{\alpha}$ then $\left(1-e^{-i \theta} z\right)^{\alpha-1} f(z)$ has the nontangential limit zero at $e^{i \theta}$ for almost all $\theta$ in $[-\pi, \pi]$.

Proof. Suppose that $\alpha>1$ and $f \in \mathscr{F}_{\alpha}$. There is a complex-valued function $g$ of bounded variation on $[-\pi, \pi]$ such that (9) holds for $|z|<1$. Since a function of bounded variation is differentiable almost everywhere, there is a set $E \subset[-\pi, \pi]$ having Lebesgue measure $2 \pi$ such that $g^{\prime}(\theta)$ exists for $\theta \in E$. If $\theta \in E$ then Theorem 3 implies $\left(1-e^{-i \theta} z\right)^{\alpha-1} f(z)$ has the nontangential limit zero at $e^{i \theta}$.

We add some remarks related to Theorem 3. Suppose that $f$ is defined by (9) where $g$ is of bounded variation and $g^{\prime}(\theta)$ exists at some $\theta$ in $[-\pi, \pi]$. Now assume that $0<\alpha<1$. The differentiability of $g$ at $\theta$ implies that there are numbers $\delta$ and $C$ such that $0<\delta<\pi, C>0$ and

$$
\left|\frac{g(\theta+t)-g(\theta)}{t}\right| \leqq C \text { for } \quad 0<|t| \leqq \delta
$$

Since $\alpha<1$ this implies that

$$
\int_{-\pi}^{\pi} \frac{|g(\theta+t)-g(\theta)|}{|t|^{\alpha+1}} d t<+\infty
$$

Therefore $f$ has a nontangential limit at $e^{i \theta}$ (see [3, Theorems 2 and 4]).

When $\alpha=1$ the assumption that $g$ is differentiable at $\theta$ does not imply that $f$ has a radial limit in the direction $\theta$. This fact is implied by (10) in Theorem 5 below. We see this implication, for example, by letting

$$
\varepsilon(r)=\frac{1}{\sqrt{\log \left(\frac{1}{1-r}\right)}}(0<r<1) .
$$

More generally, Theorem 5 asserts that Theorem 3 is sharp when $1 \leqq \alpha \leqq 2$. When $\alpha=1$ this shows that the argument given for Theorem 4 cannot be used to deduce the result: if $f \in \mathscr{F}_{1}$ then $f$ has a nontangential limit in almost all directions. This result, of course, 
is derivable from known facts about functions in $H^{p}$ spaces. We note that $\mathscr{F}_{1} \subset H^{p}$ for $0<p<1$.

The proof of Theorem 5 uses the following lemma.

Lemma 2. Let $0<\beta \leqq 1$ and let

$$
w=\frac{1}{(1-z)^{\beta}} \quad \text { where } \quad z=r e^{i \theta}
$$

If $0<r<1$ and $|\theta| \leqq 1-r$ then

$$
\operatorname{Re} w \geqq \frac{1}{2(1-r)^{\beta}} .
$$

\section{Proof.}

$$
\begin{aligned}
|1-z|^{\beta} & =\left[(1-r)^{2}+4 r \sin ^{2}\left(\frac{\theta}{2}\right)\right]^{\beta / 2} \leqq\left[(1-r)^{2}+\theta^{2}\right]^{\beta / 2} \\
& \leqq 2^{\beta / 2}(1-r)^{\beta} \leqq \sqrt{2}(1-r)^{\beta} .
\end{aligned}
$$

We may assume that $\theta>0$. Then $0<\theta<1$ and this implies

$$
\sin \theta+\cos \theta \leqq \frac{1}{1-\theta}
$$

Hence

$$
\frac{r \sin \theta}{1-r \cos \theta} \leqq \frac{(1-\theta) \sin \theta}{1-(1-\theta) \cos \theta} \leqq 1
$$

and

$$
\begin{aligned}
\cos \left[\beta \arg \frac{1}{(1-z)}\right] & =\cos \left[\beta \tan ^{-1}\left(\frac{r \sin \theta}{1-r \cos \theta}\right)\right] \\
& \geqq \cos \left[\beta \tan ^{-1}(1)\right] \geqq \cos \left(\frac{\pi}{4}\right)=\frac{\sqrt{2}}{2} .
\end{aligned}
$$

Therefore 


$$
\begin{aligned}
\operatorname{Re} w & =\frac{1}{|1-z|^{\beta}} \cos \left[\beta \arg \frac{1}{(1-z)}\right] \geqq \frac{1}{\sqrt{2}(1-r)^{\beta}} \frac{\sqrt{2}}{2} \\
& =\frac{1}{2(1-r)^{\beta}} .
\end{aligned}
$$

Theorem 5. Let $1 \leqq \alpha \leqq 2$ and suppose that $\varepsilon$ is a positive nonincreasing function on $(0,1)$ such that $\lim _{r \rightarrow 1}-\varepsilon(r)=0$. Then there is a function $f$ given by

$$
f(z)=\int_{-\pi}^{\pi} \frac{1}{\left(1-e^{-i z} z\right)^{\alpha}} d g(t) \text { for }|z|<1
$$

where $g$ is of bounded variation on $[-\pi, \pi]$ and $g$ is differentiable at 0 . Moreover, when $\alpha=1$

$$
\varlimsup_{r \rightarrow 1-} \frac{|f(r)|}{\varepsilon(r) \log \left(\frac{1}{1-r}\right)}=+\infty
$$

and when $1 \leqq \alpha \leqq 2$

$$
\varlimsup_{r \rightarrow 1^{-}} \frac{|f(r)|(1-r)^{\alpha-1}}{\varepsilon(r)}=+\infty
$$

Proof. The hypotheses on $\varepsilon$ also hold for $\sqrt{\varepsilon}$. Hence it suffices to show such an $f$ exists where (10) and (11) are respectively replaced by

$$
\varlimsup_{r \rightarrow 1-} \frac{|f(r)|}{\varepsilon(r) \log \left(\frac{1}{1-r}\right)} \geqq 1
$$

and

$$
\varlimsup_{r \rightarrow 1-} \frac{|f(r)|(1-r)^{a-1}}{\varepsilon(r)} \geqq 1
$$

Let $\left\{x_{n}\right\}(n=1,2, \ldots)$ be a strictly decreasing sequence of real numbers such that $0<x_{n}<1$ and $\lim _{n \rightarrow \infty} x_{n}=0$ and let $r_{n}=1-x_{n}$. There is a strictly decreasing sequence $\left\{a_{n}\right\}$ of a positive real numbers such that $\lim _{n \rightarrow \infty} a_{n}=0$,

$$
a_{n} \geqq \frac{1}{2} \varepsilon\left(r_{n}\right)
$$


in the case $\alpha=1$ and

$$
a_{n} \geqq(\alpha-1) \varepsilon\left(r_{n}\right)
$$

when $\alpha>1$. A real-valued function $h$ is defined on $[-\pi, \pi]$ as follows. Let $h(0)=0$ and require that $h$ is odd and on $(0, \pi]$ let $h$ be defined as described next. Let $x_{n}^{\prime}=$ $\frac{1}{2}\left(x_{n}+x_{n+1}\right)$. For $x_{1}<x \leqq \pi$ let $h(x)=a_{1}$ and for $x_{n+1}<x<x_{n}^{\prime}$ let $h(x)=a_{n+1}$. On each interval $\left[x_{n}^{\prime}, x_{n}\right]$ let $h$ be linear and let $h\left(x_{n}^{\prime}\right)=a_{n+1}$ and $h\left(x_{n}\right)=a_{n}$. Then $h$ is continuous on $(0, \pi]$ and on $[-\pi, 0)$ and since $\lim _{n \rightarrow \infty} a_{n}=0, h$ is continuous at 0 .

For $-\pi \leqq t \leqq \pi$ let $k(t)=\int_{-\pi}^{t} h(s) d s$. Then $k$ is of bounded variation on $[-\pi, \pi]$ and $k^{\prime}(t)=h(t)$ for $-\pi<t<\pi$. Let $f$ be defined by

$$
f(z)=\int_{-\pi}^{\pi} K\left(e^{-i t} z\right) d k(t)
$$

for $|z|<1$, where

$$
K(z)=\frac{z}{(1-z)^{\alpha}} .
$$

It is not difficult to show that (16) can be expressed

$$
f(z)=\int_{-\pi}^{\pi} \frac{1}{\left(1-e^{-i t} z\right)^{a}} d g(t)
$$

for $|z|<1$, where $g$ is a complex-valued function which is of bounded variation on $[-\pi, \pi]$ and is differentiable at 0 .

Equation (16) is the same as $f(z)=\int_{-\pi}^{\pi} K\left(e^{-i t} z\right) h(t) d t$. Since $h$ is odd and $K\left(e^{-i t} r\right)=$ $\overline{K\left(e^{i t} r\right)}$ for $0<r<1$ it follows that

$$
f(r)=-2 i \int_{0}^{\pi} \operatorname{Im}\left[K\left(e^{i t} r\right)\right] h(t) d t .
$$

If $0 \leqq t \leqq \pi$ and $0<r<1$ then $\operatorname{Im} K\left(e^{i t} r\right) \geqq 0$ and $h(t) \geqq 0$. Thus, (18) implies

$$
\begin{aligned}
|f(r)| & \geqq 2 \int_{0}^{\pi} \operatorname{Im}\left[K\left(e^{i t} r\right)\right] h(t) d t \geqq 2 \int_{x_{n}}^{\pi} \operatorname{Im}\left[K\left(e^{i t} r\right)\right] h(t) d t \\
& \geqq 2 a_{n} \int_{x_{n}}^{\pi} \operatorname{Im} K\left(e^{i t} r\right) d t .
\end{aligned}
$$

This inequality is the same as 


$$
|f(r)| \geqq 2 a_{n} \operatorname{Im}\left\{\int_{x_{n}}^{\pi} K\left(e^{i t} r\right) d t\right\}
$$

Suppose that $\alpha=1$. Then

$$
\begin{gathered}
\operatorname{Im}\left\{\int_{x_{n}}^{\pi} K\left(e^{i t} r_{n}\right) d t\right\}=\operatorname{Im}\left\{\int_{x_{n}}^{\pi} i \frac{d}{d t} \log \left(1-e^{i t} r_{n}\right) d t\right\} \\
=\log \frac{1}{\left|1-e^{i x_{n}} r_{n}\right|}+\log \left(1+r_{n}\right) \geqq \log \frac{1}{\left|1-e^{i x_{n}} r_{n}\right|} \\
=\frac{1}{2} \log \left[\frac{1}{\left(1-r_{n}\right)^{2}+4 r_{n} \sin ^{2}\left(\frac{x_{n}}{2}\right)}\right] \\
\geqq \frac{1}{2} \log \left[\frac{1}{\left(1-r_{n}\right)^{2}+x_{n}^{2}}\right]=\log \frac{1}{\left(1-r_{n}\right)}-\frac{1}{2} \log 2 .
\end{gathered}
$$

Therefore, (19) and (14) imply

$$
\left|f\left(r_{n}\right)\right| \geqq \varepsilon\left(r_{n}\right)\left\{\log \frac{1}{\left(1-r_{n}\right)}-\frac{1}{2} \log 2\right\}
$$

This proves (12).

Suppose that $1<\alpha \leqq 2$. Then Lemma 2 implies

$$
\begin{aligned}
\operatorname{Im}\left\{\int_{x_{n}}^{\pi} K\left(e^{i t} r_{n}\right) d t\right\} & =\operatorname{Im}\left\{\int_{x_{n}}^{\pi} \frac{i}{-\alpha+1} \frac{d}{d t}\left[\left(1-e^{-i t} r_{n}\right)^{-\alpha+1}\right] d t\right\} \\
& =\frac{1}{\alpha-1}\left\{\operatorname{Re} \frac{1}{\left(1-e^{i x_{n}} r_{n}\right)^{\alpha-1}}-\frac{1}{\left(1+r_{n}\right)^{\alpha-1}}\right\} \\
& \geqq \frac{1}{\alpha-1}\left\{\frac{1}{2\left(1-r_{n}\right)^{\alpha-1}}-\frac{1}{\left(1+r_{n}\right)^{\alpha-1}}\right\}
\end{aligned}
$$

Therefore, (19) and (15) imply

$$
\left|f\left(r_{n}\right)\right| \geqq 2 \varepsilon\left(r_{n}\right)\left\{\frac{1}{2\left(1-r_{n}\right)^{\alpha-1}}-\frac{1}{\left(1+r_{n}\right)^{\alpha-1}}\right\}
$$


This proves (13).

\section{REFERENCES}

1. L. Carleson, Selected Problems on Exceptional Sets (Van Nostrand, New York, 1967).

2. D. J. Hallenbeck and T. H. MacGregor, Growth and zero sets of analytic families of Cauchy-Stieltjes integrals, J. Analyse Math., to appear.

3. D. J. Hallenbeck and T. H. MacGregor, Radial limits and radial growth of CauchyStieltjes transforms, Complex Variables 21 (1993), 219-229.

4. J. B. Twomey, Tangential boundary behavior of the Cauchy integral, J. London Math. Soc. 37 (1988), 447-454.

Department of Mathematical Sciences

UNIVERSITY OF Delaware

NewARK

Delaware 19716, U.S.A.
Department of Mathematics and Statistics SUNY at Albany 1400 Washington Avenue

Albany, New York 12222, U.S.A. 